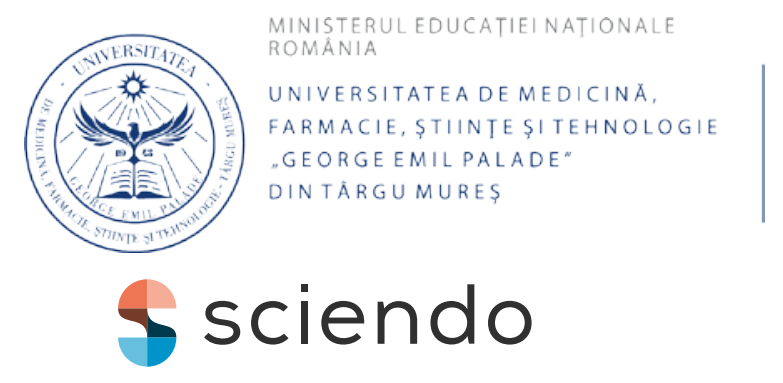

\author{
Acta Marisiensis. SeriaTechnologica \\ Vol. 17 (XXXIV) no. 2, 2020 \\ ISSN 2668-4217, ISSN-L 2668-4217
}

10.2478/amset-2020-0011

\title{
EDITORIAL: SECOND VOLUME OF ACTA MARISIENSIS. SERIA TECHNOLOGICA
}

\author{
Ildiko PETER ${ }^{1}$, Zoltan GERMAN-SALLO ${ }^{2}$, Liviu MOLDOVAN ${ }^{3}$ \\ ${ }^{1}$ University of Medicine, Pharmacy, Science and Technology" G.E. Palade" of Târgu Mureş \\ Nicolae Iorga Street, no. 1, 540088, Mureş County, Romania \\ 1'ildiko.peter@umfst.ro, ${ }^{2}$ zoltan.german-sallo@umfst.ro, ${ }^{3}$ liviu.moldovan@umfst.ro
}

\section{Welcome to the 2020 second issue of the Acta Marisiensis. Seria Technologica!}

This writing contains some update with respect to those shared in the first volume and highlights some statistics about the related topics of the current issue.

The blinded review process of at least two reviewers for all submitted work is confirmed independently if it arrives from local, domestic or foreign authors, as fortunately it often happens. In case of "Accept" recommendation, the paper will be included in the current issue or kept for further issues if the maximum number of nine contributions has already been reached. Contrarily, if the recommendation is "Reject" or it is sent back for any major or minor revision, the paper is sent back to the author(s) for revision. This can delay the publication, since reviewers are asked to check the updated version one more time. To prevent delay in publication of the outcomes of their research, potential authors are required to submit their contribution all along the year.

Reviewers are asked to indicate their opinion about the quality of the manuscript: an essay submitted by the Reviewer includes his/her decision after the quality check of the paper. The reviewers are requested to look for the (i) scope (is the manuscript appropriate for publication in the present journal?), (ii) advancement (is this an important contribution to the field?), (iii) novelty (is this new material different from earlier publications?), (iv) validity (is the research well planned and performed?), (v) data (are the data described, evaluated and deduced appropriately?), (vi) clarity (are the concepts communicated clearly?), (vii) compliance (are all ethical and journal requirements met?).

Another source of the delay is due to the submissions without considering the suggested template. Template in both Latex and Word versions will be soon available on the Journal web page.

The present volume contains 9 articles authored by researchers from:

1. Water and Water Structures Engineering Department, Faculty of Engineering, Zagazig University, Zagazig, Egypt;

2. Mechanical Engineering Department of, Integral University, Lucknow, Uttar Pradesh, India;

3. Industrial Research Institute for Automation and Measurements, Warsaw, Poland;

4. Institute for Technology and Quality. Porto Salvo, Portugal;

5. Department of Electronics and Telecommunications, Politecnico di Torino, Italy;

6. National Institute for Research \& Development in Electrical Engineering ICPE-CA, Bucharest, Romania;

7. Faculty of Materials Engineering and Mechanics, Valahia University of Targoviste, Targoviste, Romania

8. Faculty of Physics, University of Bucharest, Romania;

9. Technical University of Cluj-Napoca, Romania; 
10. University of Medicine, Pharmacy, Science and Technology" G.E. Palade" of Târgu Mureş.

The present issue will go with a Supplementary Volume, including some student contributions. Actually, it will be a good opportunity to award the best research work of such Student contributions, coming up shortly.

During the time, the above-mentioned actions and other constructive arrangements will be applied, to provide an as clear as possible image about the academic life of our community.

Any feedback from our research community is more than welcome. Receive any your input targeting to reach the above-mentioned aims that we believe will be of benefit of our whole community is more than appreciated.

\section{Contributions in a nutshell... (Instead of the list of contents)}

The influence of the sintering temperature on both the structure and hysteresis loops of iron ions doped $\mathrm{Pb}($ ZrxTi1-x $) \mathrm{O}_{3}$ system has been investigated in "EFFECTS OF SINTERING TEMPERATURE ON STRUCTURAL AND ELECTRICAL PROPERTIES OF IRON DOPING PZT CERAMICS" by A. I. Dumitru, F. Clicinschi, T.G. Dumitru, D. Patroi, J.ana Pintea, G. Velciu, I. Peter. Three compositions and two different sintering temperature have been considered; the ceramic materials have been obtained by solid-state reaction technique and then have been experimentally characterized. The main outcomes of the study consists in the laboratory demonstration of the fact that the compositions have a similar behavior with a "hard" PZT conventional ceramic one.

In the paper entitled "BUCKLING ANALYSIS OF INNOVATIVE CORRUGATED COLUMN BY USING RESPONSE SURFACE METHODOLOGY" by Mohd Reyaz Ur RAHIM1, Prem Kumar BHARTI, the authors describe the effect of variation in the sectional properties like mean diameter, thickness, amplitude, and frequency of corrugation on the buckling axial load. A quadratic model was developed to correlate the independent variables for maximum buckling load by using central composite design method and a statistical tool based analysis was carried out to compare the different combinations in order to find the most important factor on buckling load.

Other topic, a very "hot" one in this period, is related to a study focusing on the real picture about the modular online education at the Faculty of Engineering and Information Technology of UMFST "G.E.Palade" of Tg.Mures. The purpose is to identify the deficiencies of the problems raised by the technical disciplines that require practical applications in laboratory classes. Results of the study based on a questionnaire addressed for both the students and the lecturers is presented by $M$.
Bucur and B. Bucur, in the paper entitled "ASPECTS REGARDING THE ONLINE MODULAR EDUCATION SYSTEM COMPARED WITH THE CLASSICAL ONSITE SYSTEM". Furthermore, some new directions for the development of modular online education have been identified, as alternative solution to the onsite classic education.

M. Bogdan proposes a generalized solution of a Cauchy problem given by a nonhomogeneous linear differential system. The paper entitled "A NOTE ON GENERALIZED SOLUTION OF A CAUCHY PROBLEM GIVEN BY A NONHOMOGENEOUS LINEAR DIFFERENTIAL SYSTEM" considers the case of the free term having at most countable number of discontinuity points.

L. Carosso, L. Mattiauda, M. Allegretti in the paper entitled "A SURVEY ON DEVICES EXPLOITING LORA COMMUNICATION" highlight that Information and Communication Technologies have experienced a great application in many fields, such as smart homes, health monitoring, environmental monitoring. According to this paper, it is expected that the Internet of Things will become increasingly prevalent in our normal life. Because of the low power consumption, LoRa is one of the best suitable protocol for these applications

In the paper entitled "THE INTEGRAL FORM OF THE SCHUR INEQUALITY” by B. Finta, the integral form of the original Schur inequality is shown and some applications are discussed.

The paper entitled "MODELING IN WATER RESOURCES MANAGEMENT IN EAST NILE DELTA: Review" authored by F. Heba Abdelwaha, E M. Ramadan, M. N. Abdelazim shows that providing a safe water supply for all countries constitutes one of the key challenges of the twenty-first century. The review is limited to surface water, but groundwater has been inexplicitly included. The paper concludes that by planning and by managing the problems it will be possible to identify some solutions and cover the gap between water supply and demands, and to guide future researches on water.

J. Zielinski, R. Almeida, L. Moldovan, in the paper entitled "LEGISLATION REVIEW AND POLICY RECOMMENDATION FOR WORK-BASED LEARNING" focalizes to review the legislation framework in the field of work-based learning (WBL), conducted in six European countries: Romania, Poland, Portugal, Germany, Bulgaria and Cyprus, and to identify the policies and the legal framework set, or lack of such, in the field of WBL through a comparative analysis between formal regulations. Finally, policy recommendations for performing of evidence-based policy in the field of WBL are formulated.

Z. German Sallo, Z. German Sallo, Jr. in the paper entitled "METHODS IN COMPLEXITY ANALYSIS OF DISCRETE TIME SIGNALS" uses information theory- 
based estimates of complexity as different types of entropies in order to estimate the regularity of various synthesized discrete time signals. Discrete time signals carry information about systems and their internal functional mechanisms, therefore their complexity analysis is important. Results show that this kind of indices can be a useful tool in systems diagnostic, fault detection and further development.

Here we are remembering the authors about the reward scheme put in force this year. The prizes of the categories publicized in the previous issues will be announced in the first issue of the next year. The editorial board will do its best to identify high profile members for the judge commission(s).

With the approaching holiday, we wish to all our Readers all the best season Greetings, health and full satisfaction at both personal and professional level! 